\title{
Pressure-Induced Tunable Electron Transfer and Auger Recombination Rates in CdSe/ZnS Quantum Dot-Anthraquinone Complexes
}

Huifang Zhao ${ }^{a, \dot{\dagger}}$, Hang Yin ${ }^{a, \dagger}$, Xiaochun Liu ${ }^{a}$, Hui Li ${ }^{a}$, Ying Shi ${ }^{a, *}$, Cailong Liu ${ }^{a, *}$, Mingxing Jin ${ }^{a}$, Jianbo Gao ${ }^{b, *}, Y_{i}$ Luo $^{c}$ and Dajun Ding ${ }^{a, *}$

anstitute of Atomic and Molecular Physics, Jilin University, Changchun 130012, China.

bUltrafast Photophysics of Quantum Devices Laboratory, Department of Physics and Astronomy, Clemson University, South Carolina, 29634, USA.

${ }^{\mathrm{c}}$ Hefei National Laboratory for Physical Sciences at the Microscale, University of Science and Technology of China, Hefei, Anhui 230026, China.

$\dagger$ These authors contributed equally to this work.

\section{Corresponding Author}

*E-mail: shi_ying@jlu.edu.cn (Y.S.)

*E-mail: cailong_liu@jlu.edu.cn (C.L.)

*E-mail: jianbogao.nano@gmail.com (J.G.)

*E-mail: dajund@jlu.edu.cn (D.D.) 
Table S1. The conduction band (CB) and the valence band (VB) of CdSe/ZnS which are obtained from cyclic voltammetry measurements, and the band energy of LUMO, HOMO potentials of AQ acceptor molecules which are calculated by density functional theory.

\begin{tabular}{cccccc}
\hline System & CB (eV) & VB (eV) & LUMO (eV) & HOMO (eV) & Wavelength (nm) \\
\hline CdSe/ZnS & -2.380 & -5.409 & & & 535.0 \\
AQ & & & -3.142 & -7.367 & 293.4 \\
\hline
\end{tabular}

(a)

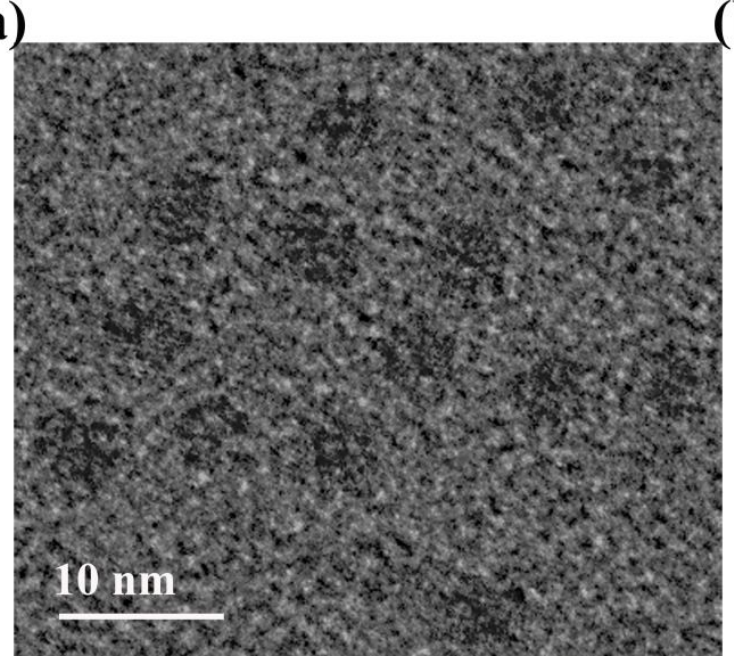

(b) $\operatorname{Energy~(eV)}$

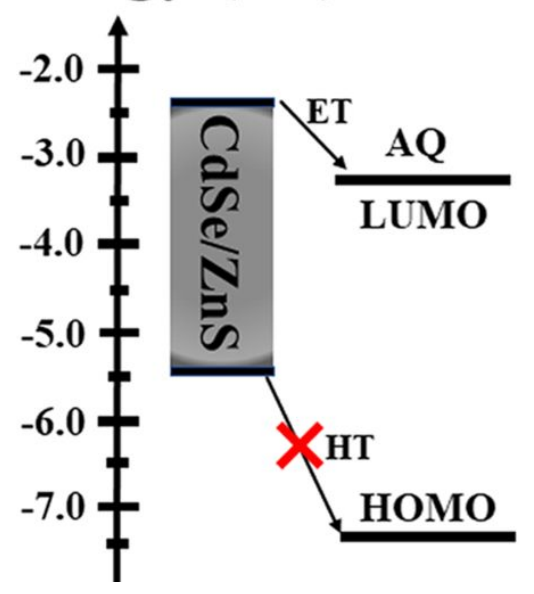

Figure S1. (a) TEM (transmission electron microscope) image of the CdSe/ZnS used in our experiments with an approximately $5 \mathrm{~nm}$ in diameter (scale bar, $10 \mathrm{~nm}$ ). (b) Energy diagram of the ET process. 
Table S2. The peak positions of 1S bleach signal at different pressures. A clear blue shift exists from 1 atm to $2.4 \mathrm{GPa}$.

\begin{tabular}{cc}
\hline Pressure & Peak Position $(\mathrm{nm})$ \\
\hline $1 \mathrm{~atm}$ & 530 \\
$0.1 \mathrm{GPa}$ & 528 \\
$0.2 \mathrm{GPa}$ & 527 \\
$0.3 \mathrm{GPa}$ & 525 \\
$0.5 \mathrm{GPa}$ & 524 \\
$0.7 \mathrm{GPa}$ & 519 \\
$0.9 \mathrm{GPa}$ & 516 \\
$1.1 \mathrm{GPa}$ & 515 \\
$1.3 \mathrm{GPa}$ & 513 \\
$1.6 \mathrm{GPa}$ & 512 \\
$2.4 \mathrm{GPa}$ & 510 \\
\hline
\end{tabular}




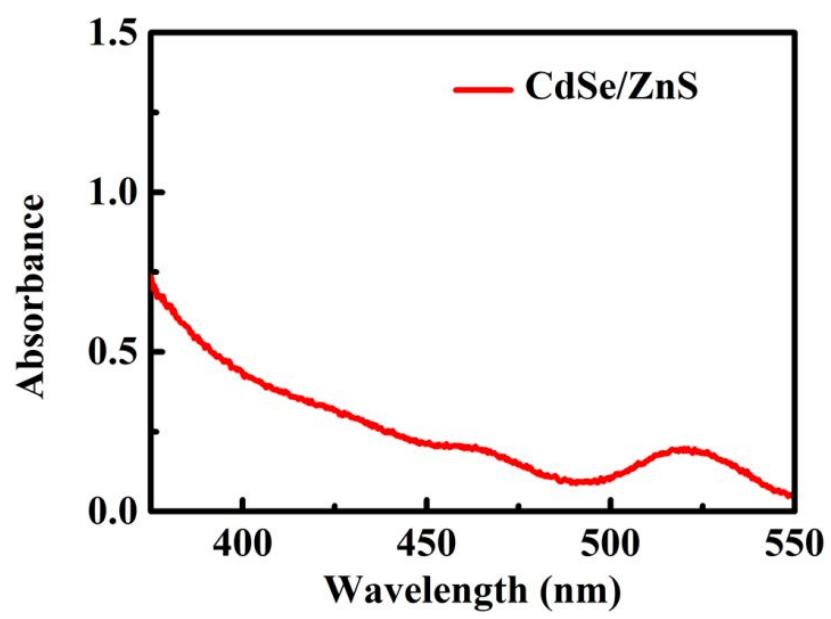

Figure S2. Steady-state spectral absorption of the QDs in CHX at atmospheric pressure. 
Table S3. Lifetimes for ET and AR processes at different pressures. $\tau_{1}$ and $\tau_{2}$ stand for ET and AR processes respectively. The meaning of the proportion is the deviation percentage.

\begin{tabular}{ccc}
\hline Pressure & $\tau_{1}(\mathrm{ps})$ & $\tau_{2}(\mathrm{ps})$ \\
\hline $1 \mathrm{~atm}$ & $3.500 \pm 0.886(25.31 \%)$ & $549.0 \pm 105.0(19.13 \%)$ \\
$0.1 \mathrm{GPa}$ & $1.179 \pm 0.162(13.74 \%)$ & $560.0 \pm 98.70(17.62 \%)$ \\
$0.2 \mathrm{GPa}$ & $1.280 \pm 0.155(12.10 \%)$ & $590.0 \pm 145.0(24.57 \%)$ \\
$0.3 \mathrm{GPa}$ & $1.397 \pm 0.179(12.81 \%)$ & $624.6 \pm 126.0(20.17 \%)$ \\
$0.5 \mathrm{GPa}$ & $0.700 \pm 0.215(30.71 \%)$ & $606.3 \pm 152.0(25.07 \%)$ \\
$0.7 \mathrm{GPa}$ & $0.860 \pm 0.140(16.27 \%)$ & $625.4 \pm 181.0(27.74 \%)$ \\
$0.9 \mathrm{GPa}$ & $0.980 \pm 0.293(29.89 \%)$ & $646.8 \pm 229.0(35.40 \%)$ \\
$1.1 \mathrm{GPa}$ & $0.435 \pm 0.125(28.73 \%)$ & $622.8 \pm 235.1(37.74 \%)$ \\
$1.3 \mathrm{GPa}$ & $0.490 \pm 0.0856(17.46 \%)$ & $631.7 \pm 126.0(19.94 \%)$ \\
$1.6 \mathrm{GPa}$ & $0.527 \pm 0.119(22.58 \%)$ & $640.7 \pm 194.0(29.99 \%)$ \\
$2.4 \mathrm{GPa}$ & $0.598 \pm 0.128(21.40 \%)$ & $660.8 \pm 173.0 .(26.25 \%)$ \\
\hline
\end{tabular}

